\title{
Paper
}

\section{Haptic Interface with a Stylus for a Mobile Touch Panel}

\author{
Shingo Nagasaka (student member) ${ }^{\dagger}$, Yuki Uranishi ${ }^{\dagger \dagger}$, Shunsuke Yoshimoto $^{\dagger}$, \\ Masataka Imura $^{\dagger}$, Osamu Oshiro ${ }^{\dagger}$
}

\begin{abstract}
This paper proposes a system that provides the sensation of touching virtual objects in a mobile touch panel using a retractable stylus and the mobile touch panel. The proposed system provides a sensation like the stylus is being inserted into the monitor, and that the user is actually touching the object in the screen when the user pushes a retractable stylus downward on the display. A DC motor is mounted in the retractable stylus, and this motor shrinks the length of the stylus based on feedback from a pressure sensor in the tip of the stylus. When the tip of the virtual stylus touches a virtual object, a voice coil motor in the stylus oscillates according to the surface of the virtual object. So the user experiences a sensation like touching the object on the monitor by using the proposed system.
\end{abstract}

Key words: virtual reality, haptics, stylus, tablet PC, touch panel

\section{Introduction}

People recognize the shape, texture, and temperature of an object by touching it. People, ranging from surgeons to carpenters, work with the aid of their sense of touch of the objects that they are handling when performing complex or sensitive tasks. To improve their skills, there is no other way but on-the-job practice and experience of a haptic sensation.

Several systems have been proposed recently that provide a haptic sensation to a user via virtual reality (VR) technology ${ }^{1}$. VR is a technology that enables humans to perceive a realistic environment virtually via artificial stimulus applied to the sensory organs. It is desirable that the VR systems simulate and give the same sensation as the actual one to the user. Some VR systems provide visual information to a user via a head-mounted display (HMD) $)^{2}$, and provide acoustic information via headphones ${ }^{3)}$. Similarly, some VR systems provide the sensation of touching an object via a haptic display. Haptic displays are devices that give a tactile and a force sensation to the user artificially. These devices enable users to experience the sensation of touching virtual objects. However, systems using conventional devices are very large and complicated for the average user.

To remedy this, we propose a system that gives a

\footnotetext{
Received March 19, 2015; Revised July 17, 2015; Accepted August 21, 2015

$\dagger$ Osaka University

(Osaka, Japan)

$\dagger \dagger$ Kyoto University

(Kyoto, Japan)
}

haptic sensation to a user simply by using a stylus and a mobile touch panel that includes a tablet computer. Figure 1 shows the concept of the system proposed in this paper. The proposed system enables users to interact with a virtual object behind the touch panel. When the user presses the stylus against the touch panel, the stylus shrinks in length according to the pressing force. The proposed system shows an image as if the stylus had entered into the touch panel, according to the shrinkage of the stylus. When the virtual tip of the stylus touches the virtual object, the stylus provides a virtual haptic sensation from the object to the user by shrinking and oscillating itself. The system also estimates the viewpoint of the user from images received from a camera in the top of the touch panel and displays the virtual image according to that viewpoint. Conventional haptic devices are fastened in workspace, and a user should stay in front of the devices to experience haptic VR. On the other hand, the proposed system enables the user to carry the haptic device and experience the haptic VR in a mobile use, such as mobile visuo-haptic augmented reality.

This paper describes a theory to realize the proposed system. This paper is organized as follows: In Section 3 , the concept of the proposed system is outlined. Section 4 describes the proposed method for transmitting a haptic sensation. In Section 5, a method for detecting the viewpoint of the user and the angle between the touch panel and the stylus is introduced. The two experimental results obtained in Section 6 show that the proposed system with haptic feedback is effective in enabling users to experience a haptic feeling of the virtual 


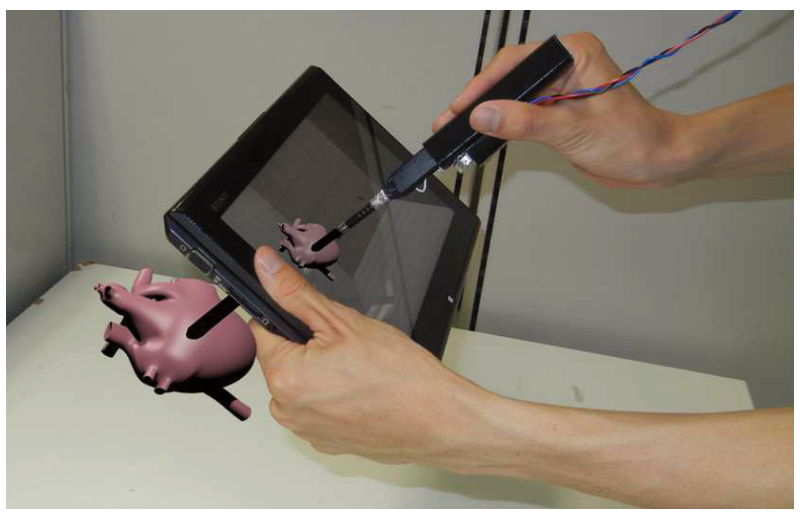

Fig. 1 Concept of the proposed system.

object.

\section{Related Works}

Section 2. 1 describes general haptic devices, and Section 2.2 describes techniques using a modern stylus for a touch screen.

\subsection{Conventional Haptic Devices}

Several devices for haptic VR have been proposed that give users the feeling of touching virtual objects according to the actions of the users ${ }^{4}$.

$\mathrm{PHANTOM}^{5)}$ is a representative stationary haptic device, consisting of a pen-like grip, a base, and a jointed arm that connects the first two components. PHANToM senses the 6 degree-of-freedom (DoF) position of the grip and gives 3 DoF force feedback to the user. PHANToM provides force feedback to a user touching an imaginary object, such as a soft sphere, a flat wall, a sharp corner or an irregular wall. However, the user is restrained by the device because PHANToM must be sited in a fixed location to provide force feedback.

Another representative stationary device is CyberGrasp $^{6)}$. CyberGrasp is a glove type of force feedback system. The user wears an exoskeleton fitted over a data glove, and CyberGrasp provides resistive force feedback to each finger. CyberGrasp enables the user to feel the size and the shape of virtual objects and grasp them.

When using these devices to construct a VR system with a conventional monitor, the user may get an uncomfortable feeling because of a disagreement between the point of touch of the virtual object shown on a touch panel and the point on the device where the user feels feedback. To solve this problem, HMDs are frequently used. However, using HMD complicates the system, and it is not possible to use this system everywhere.

\section{2 Modern Stylus for a Touch Panel}

Several systems for human-computer interaction using a touch screen and a stylus have been proposed. Please note that none of these systems contain haptic devices of the sort mentioned above, and the systems referred to in this section are not intended to provide a haptic sensation to a user. However, the systems do enable users to "insert themselves" into the touch panel by using a retractable stylus.

Beyond $^{7)}$ consists of a retractable stylus, a table-top display, infrared markers, and cameras set up in an environment. A virtual tip to the stylus is rendered when the retractable stylus is pushed against a table-top display. The head position of the user is detected by the infrared markers and the cameras, and the virtual objects and the tip of the stylus are rendered correctly according to the head position. The system enables the user to interact with a virtual object under the table. However, the system is stationary due to the need to set up sensors in the environment. In addition, the stylus neither shrinks nor extends actively because the stylus does not have any actuators, such as a motor. Therefore, the user is unable to experience a haptic sensation from the virtual object.

ImpAct ${ }^{8)}$ is another novel interface using a smartphone and a retractable stylus. The force feedback is represented by simply stopping the shrinkage of the stylus. However, the system gives only rigid force feedback. In addition, the system does not provide a tactile sensation from the virtual object. Moreover, it does not consider the viewpoint of the user. When using a smartphone, the user moves her/his viewpoint constantly. Therefore, it is necessary to detect the viewpoint in order to present a natural-looking image to the user.

In this study, our aim is to construct a system that enables a user to experience the feeling of touching virtual objects without using devices fixed in one location in an environment. We propose a mobile system for providing haptic and visual information from a virtual object. The proposed system enables the user to touch virtual objects behind a mobile touch panel and perceive the surface texture of the object.

\section{Proposed System}

\section{1 Concept of the Proposed System}

The proposed system consists of a retractable stylus, a camera, and a mobile touch panel. Figure 2 shows a scene in which a user is touching a virtual heart be- 


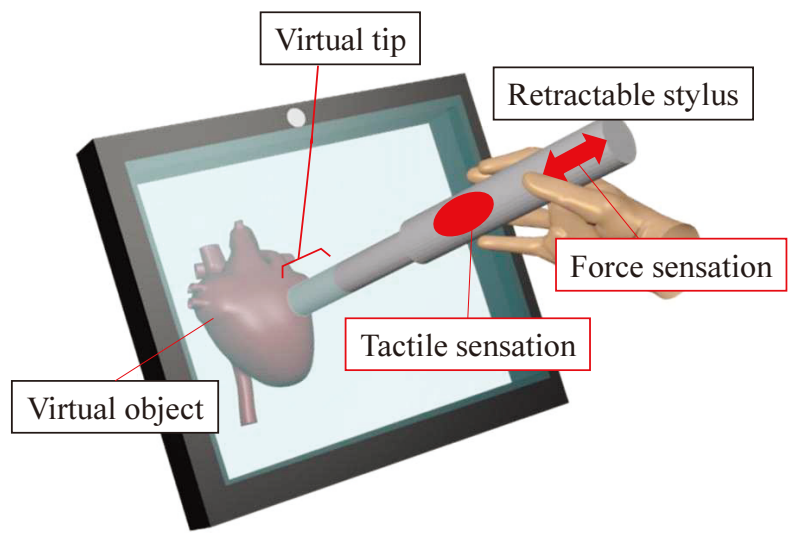

Fig. 2 Overview of the proposed system.

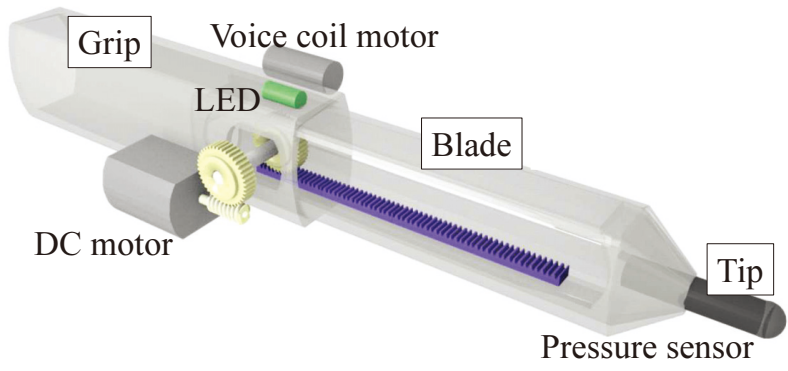

Fig. 3 Structure of the retractable stylus.

hind a mobile touch panel and is receiving a reaction force and a tactile sensation from the virtual heart. The viewpoint of the user is estimated from an image taken by a camera positioned at the top of the touch panel. The system shows a virtual space behind the panel surface, and the user can interact with virtual objects using the retractable stylus.

\section{2 Structure of the Retractable Stylus}

Figure 3 shows the structure of the stylus proposed in this paper. The stylus consists of a grip, a blade, and a tip. The user holds the stylus by the grip. A DC motor in the stylus is used to shrink and extend the stylus. A worm gear is attached to the shaft of the DC motor. A pressure sensor is fitted to the tip of the stylus to measure the pressing force applied by the user. A voice coil motor is attached to the grip to give the user the tactile sensation of the surface of the object by oscillating.

\section{Elasticity and Texture Modeling}

Haptic devices need to be fixed to a point in space to provide force feedback to a user. In our proposed system, the system gives force feedback to the user as the tip of the stylus acts as a fulcrum while the tip is touching the touch panel. Figure 4 shows a model for providing haptic feeling in the proposed system. The

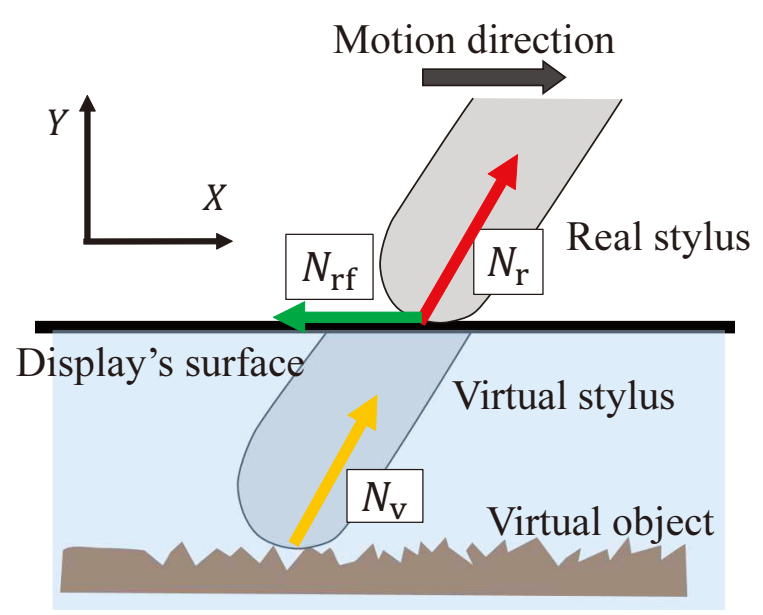

Fig. 4 Model for haptic feeling.

proposed system simulates a virtual force $N_{\mathrm{v}}$ from the virtual object behind the touch panel and gives the real force $N_{\mathrm{r}}$ to the user instead of $N_{\mathrm{v}}$. The origin of the $N_{\mathrm{r}}$ is the center of the tip, and the origin of $N_{\mathrm{v}}$ is the point where the virtual tip touches the panel. The force feedback from the touch panel is a product of the force applied in the direction of the shrinkage of the stylus $N_{\mathrm{r}}$ and the friction force parallel to the panel surface $N_{\text {rf }}$. The friction force $N_{\text {rf }}$ is in the opposite direction to the motion of the stylus. The friction force changes according to the frequency of vibration ${ }^{9)}$. This indicates that changing the frequency of a vibration controls $N_{\text {rf }}$. Shrinking the stylus provides the macroscopic force from the virtual objects, and the oscillation of the voice coil motor provides the microscopic force from the texture on the surface of the virtual objects. The DC motor drives to change the length of the stylus. When the stylus is not touching the monitor, the value of the pressure sensor is 0 . When the tip of the stylus touches the monitor, the stylus shrinks according to the value received from the pressure sensor.

\section{1 Modeling of Feedback Force}

When the tip of the stylus touches the touch panel, the DC motor drives and shrinks the stylus. The proposed system considers the force in the same direction as the direction of the shrinkage of the stylus. Figure 5 shows the relationship between the force from the stylus and the touch panel, and the relationship between the real force and the virtual force. $F_{\mathrm{u}}, F_{\mathrm{m}}$, and $N_{\mathrm{r}}$ are the force from the user, the force from the motor, and the force from the touch panel, respectively. $N_{r}$ is measured by the pressure sensor. If $F_{\mathrm{u}}-F_{\mathrm{m}}<0$, the stylus extends. Conversely, if $F_{\mathrm{u}}-F_{\mathrm{m}}>0$, the stylus shrinks. If $F_{\mathrm{u}}=F_{\mathrm{m}}$, the stylus neither extends nor shrinks. The 


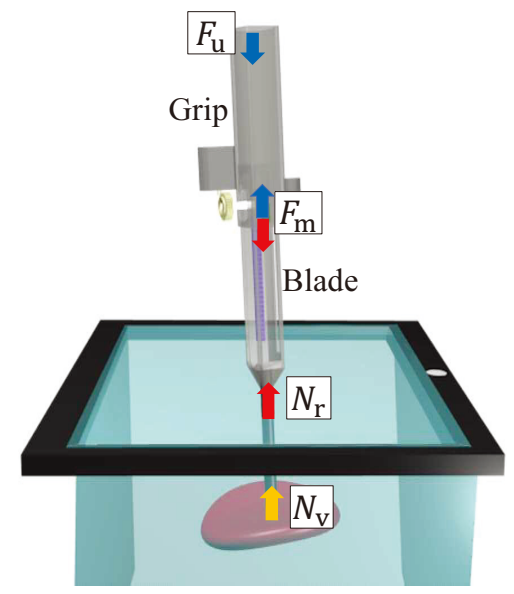

Fig. 5 Relationship between force on the stylus and force on the touch panel.

DC motor is activated with the force $\delta F_{\mathrm{m}}$ given by

$$
\delta F_{\mathrm{m}}=N_{\mathrm{v}}-N_{\mathrm{r}}
$$

Pulse width modulation (PWM) control is utilized in the proposed system. The duty ratio is defined as $D$. The maximum torque of the motor is $\tau$, and the radius of the gear attached to the motor is $r_{\mathrm{m}}$. Duty rate is given by

$$
D=\frac{\delta F_{\mathrm{m}} r_{\mathrm{m}}}{\tau} .
$$

\section{2 Calculation of the Tactile Sensation}

The proposed system transmits a tactile sensation from a virtual object by oscillating the voice coil motor. When the virtual tip of the stylus touches the virtual object behind the touch panel, the voice coil motor oscillates at a frequency according to the texture of the surface of the object.

\section{Detection of Viewpoint and Stylus by Camera}

This section describes a method for detecting the viewpoint of the user, as well as the angle formed by the stylus and touch panel surface. The viewpoint and the angle change constantly while a user is manipulating a tablet computer. To provide a realistic sensation to the user, the proposed system translates the camera output image according to the viewpoint and the angles. The proposed system uses the camera on the front face of a standard tablet computer, and a fisheye lens is added to convert the angle of view. Figure 6 shows an image taken by the frontal camera. The viewpoint of the user and the angle between the stylus and

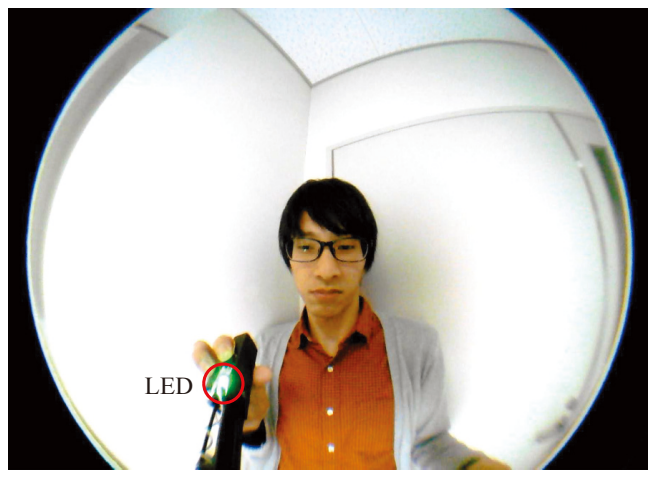

Fig. 6 Image taken by camera with fish-eyes lens.

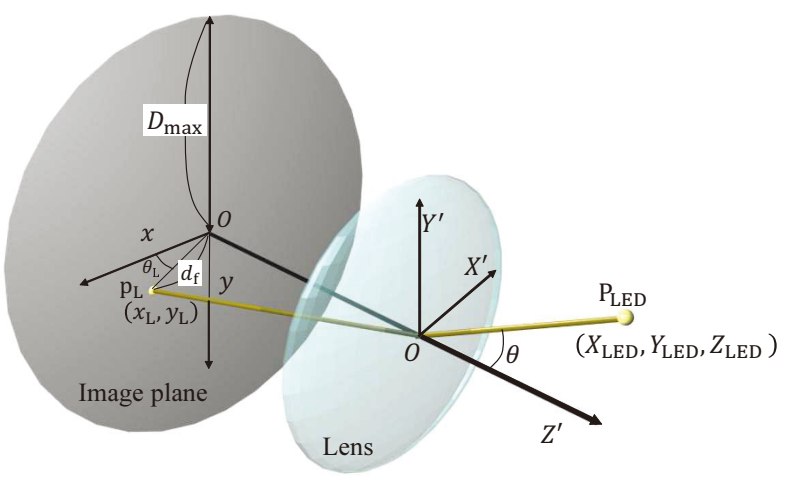

Fig. 7 Model of the fisheye lens.

the touch panel surface are estimated from the camera image.

\section{1 Estimation of Viewpoint}

First, the face of the user is detected based on Haarlike features ${ }^{11)}$. The center of gravity of the face is regarded as the two-dimensional viewpoint of the user. In addition, it is assumed that the distance between the two-dimensional viewpoint and the touch panel surface is constant.

\subsection{Estimation of Angle Formed by Stylus and Touch Panel}

To measure the angle formed by the stylus and the touch panel, a light emitting diode (LED) is attached to the stylus, and a fisheye lens is attached to the camera on the mobile touch panel. The angle formed by the stylus and the touch panel is based on the position of the LED in the captured image.

Figure 7 shows a model of the fisheye lens used. An equidistant fisheye lens is used in the proposed system. In equidistant projection, the distance from the center of the image $d_{\mathrm{f}}$ is proportional to the angle from the optical axis $\theta$. $\theta$ is calculated as

$$
\theta=k d_{\mathrm{f}},
$$

where $k$ is a proportionality factor defined by the maximum incident angle of the radius of the image plane. 


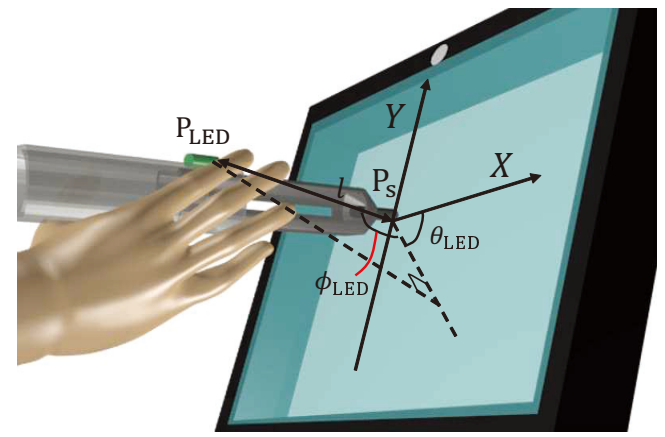

Fig. 8 Relationship between monitor and stylus.

Let the maximum incident angle be $\theta_{\max }$, and let the radius of the image plane be $D_{\max }$. Therefore, $k$ is obtained as

$$
k=\frac{\theta_{\max }}{D_{\max }} .
$$

The position of the LED $\mathrm{P}_{\mathrm{LED}}\left(X_{\mathrm{LED}}, Y_{\mathrm{LED}}, Z_{\mathrm{LED}}\right)$ in Figure 8 is obtained as

$$
\left(\begin{array}{c}
X_{\mathrm{LED}} \\
Y_{\mathrm{LED}} \\
Z_{\mathrm{LED}}
\end{array}\right)=q\left(\begin{array}{c}
\sin \theta \cos \theta_{L} \\
-\sin \theta \sin \theta_{L} \\
\cos \theta
\end{array}\right) .
$$

As Fig. 8 shows, let the distance between the point of the tip $\mathrm{P}_{\mathrm{S}}\left(X_{\mathrm{s}}, Y_{\mathrm{s}}, Z_{\mathrm{s}}\right)$ and $\mathrm{P}_{\text {LED }}$ be considered as $l$. $l$ is obtained from the shrinkage of the stylus estimated by the pressure sensor. Therefore, $\mathrm{P}_{\text {LED }}$ is estimated as

$$
\begin{array}{r}
\left(X_{\mathrm{LED}}-X_{\mathrm{S}}\right)^{2}+\left(Y_{\mathrm{LED}}-Y_{\mathrm{s}}\right)^{2}+\left(Z_{\mathrm{LED}}-\right. \\
\left.-Z_{\mathrm{s}}\right)^{2} \\
=l^{2}
\end{array}
$$

Two solutions are obtained by substituting Eq. (5) into Eq. (6). $P_{\text {LED }}$ is always in front of the touch panel. Therefore, the point that is $Z_{\mathrm{LED}}<0$ is regarded as $\mathrm{P}_{\text {LED }}$. Finally, the angles $\phi_{\text {LED }}$ and $\theta_{\text {LED }}$ are obtained as

$$
\begin{aligned}
& \phi_{\mathrm{LED}}=\sin ^{-1} \frac{Z_{\mathrm{SL}}}{l}, \\
& \theta_{\mathrm{LED}}=\left\{\begin{array}{cll}
\cos ^{-1} \frac{X_{\mathrm{SL}}}{l} & \text { if } & Y_{\mathrm{SL}} \leqq 0 \\
2 \pi-\cos ^{-1} \frac{X_{\mathrm{SL}}}{l} & \text { if } & Y_{\mathrm{SL}}>0,
\end{array}\right.
\end{aligned}
$$

where $\overrightarrow{\mathrm{P}_{\mathrm{S}} \mathrm{P}_{\mathrm{LED}}}=\left(X_{\mathrm{SL}}, Y_{\mathrm{SL}}, Z_{\mathrm{SL}}\right)$.

Table 1 Specifications of the computer.

\begin{tabular}{|c|c|}
\hline OS & Microsoft Windows 8 \\
\hline CPU & Intel Core i5-3317U $1.7 \mathrm{GHz}$ \\
\hline Memory & $4.0 \mathrm{~GB}$ \\
\hline LCD touch panel & Capacitance method TFT color liquid crystal \\
\hline Display Resolution & $1920 \times 1080$ pixels \\
\hline Webcamera & Exmor $\mathrm{R}$ for PC $(2.07$ million pixels $)$ \\
\hline
\end{tabular}

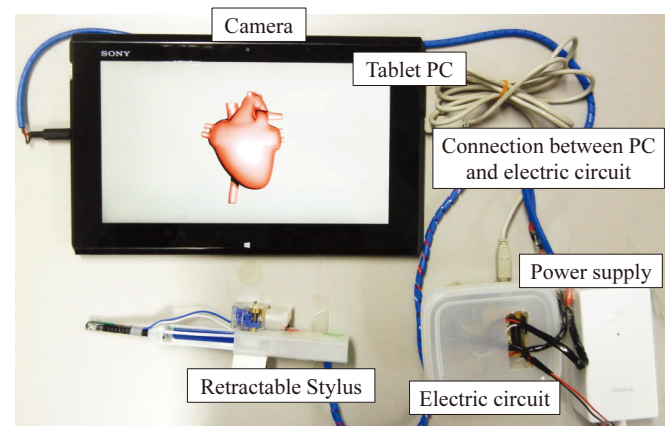

Fig. 9 Prototype of the proposed system.

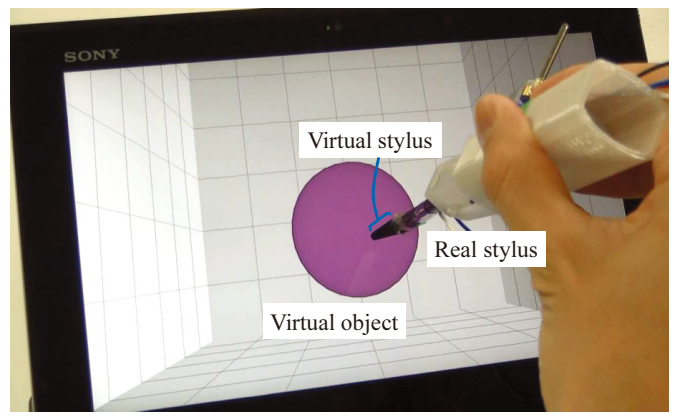

Fig. 10 Usage example of the proposed system.

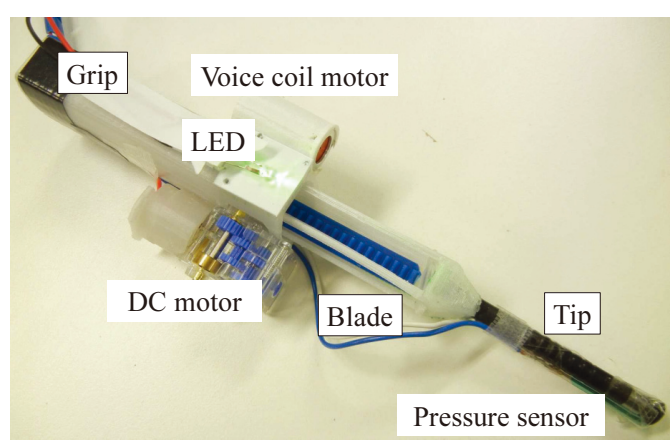

Fig. 11 Manufactured stylus.

\section{Experimental Results}

A prototype of the proposed system was implemented. Figure 9 shows an overview of the prototype. Table 1 shows the specifications of the computer used in the prototype. Figure 10 shows a usage example of the system. Figure 11 shows an implemented stylus, and Table 2 shows the specifications of the stylus components.

We conducted two experiments to ascertain the accuracy of the system and to prove the utility of the haptic presentation. The experiment has been approved by the Ethical Committee of the Graduate School of Engineering Science (25-10), Osaka University, Japan.

Table 2 Specifications of the components.

\begin{tabular}{|c|c|}
\hline DC motor & ITEM 70189 (TAMIYA) \\
\hline Voice coil motor & Haptuator (TactileLabs) \\
\hline Pressure sensor & FSR-400 (Interlink Electronics) \\
\hline
\end{tabular}




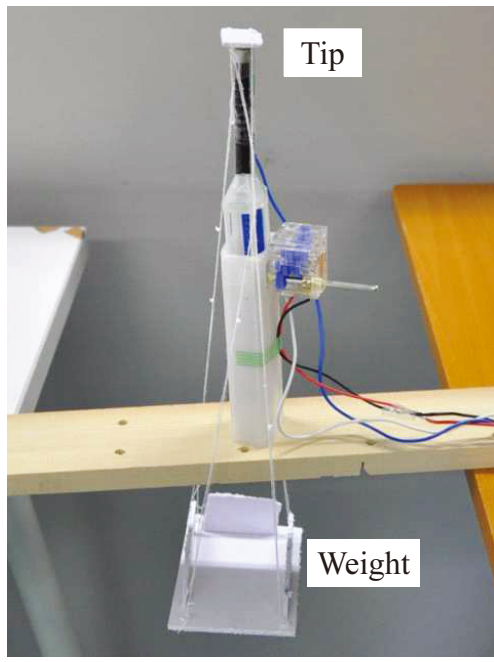

Fig. 12 Experimental setup.

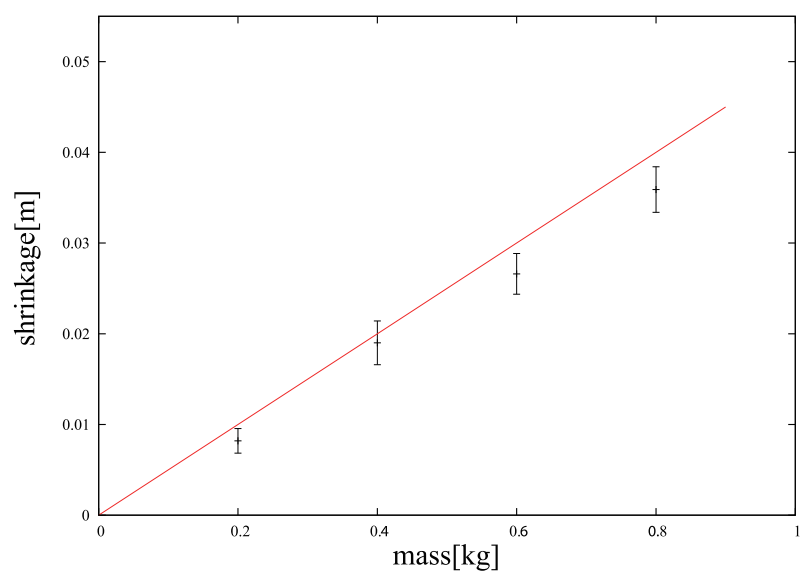

Fig. 13 Relationship between a mass of the weight and a shrinkage.

\section{1 Specifications of Manufactured Stylus}

The purpose of this experiment is to evaluate the accuracy of the shrinkage of the stylus against the force applied to the tip of the stylus. The stylus is positioned so that the tip is facing upward in the vertical direction, and weights are placed in a container underneath the stylus. We measure the shrinkage of the stylus five times. Figure 12 shows the experimental setup. Force feedback from a virtual object is assumed to be elastic force $F_{\mathrm{v}}=k x$ in proportion to spring constant $k$, which is $20 \mathrm{~N} / \mathrm{m}$. Figure 13 shows the experimental results. The black line is the measured value, and the red line is the theoretical value.

The smaller the amount of shrinkage compared to the theoretical value, the larger the mass of the weights. This is because a larger mass of weights corresponds to a larger energy loss in the pressure sensor and gear.

\section{2 Subjective Experiment}

A subjective experiment was performed to evaluate the usability of the proposed system. The purpose of

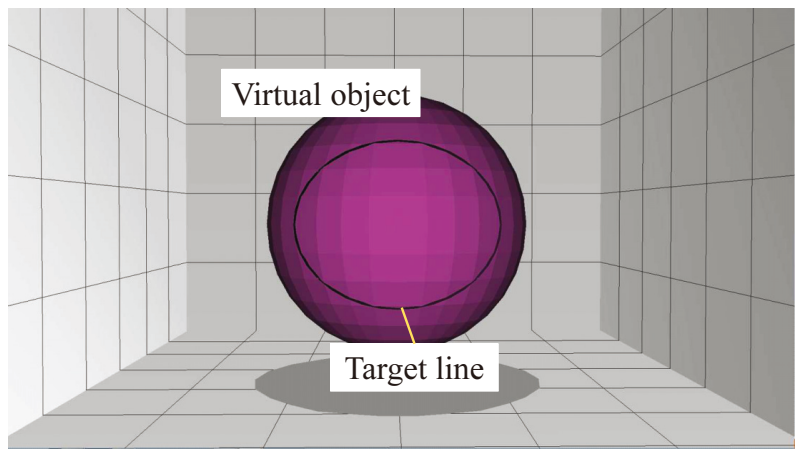

Fig. 14 Test object (sphere).

the experiment was to prove the utility of the haptic presentation in our system. In the experiment, seven subjects traced lines on two types of virtual objects using the prototype as shown in Figs. 14 and 15. Three trials were conducted: without feedback, with only force feedback, and with both force and tactile feedback. First, each subject traced the target line on the sphere shown in Fig. 14, then traced the target line on the cuboid shown in Fig. 15.

In the trial without feedback, the subject did not experience force feedback or tactile sensation, and the virtual tip of the stylus penetrated the surface of the virtual object. In the trial with only force feedback, the subject experienced only force feedback from the virtual object when the tip of the stylus touched the surface of the virtual object. However, the subject did not receive any tactile information. In the trial with force feedback and tactile feedback, the subject was able to experience force feedback and tactile feedback. When the tip disengages the target line, the voice coil motor oscillate at a constant frequency, so the user knows whether the target line is being traced correctly.

The error $R$ is defined as

$$
R=\frac{1}{M} \sum_{i=1}^{M}\left|\mathbf{X}_{\mathbf{i}}-\mathbf{P}_{\mathbf{i}}\right|,
$$

where $M$ is the number of points used to evaluate the error. $\mathbf{X}_{\mathbf{i}}$ is the position of the point sampled from lines traced by the subject, and $\mathbf{P}_{\mathbf{i}}$ is the position corresponding to $\mathbf{X}_{\mathbf{i}}$ on a correct shape. Figure 16 shows the average errors for the target lines. Statistically significant differences $(p<0.05$ with SteelDwass test) are marked above with a bar and ${ }^{*}$ symbol. A Kruskal-Wallis test was performed to analyze a difference between the trials, and there was a significant difference between the trials in case of both objects, sphere $(H=12.141, \mathrm{df}=2, p<0.005)$ and cuboid $(H=15.641, \mathrm{df}=2, p<0.005)$. 


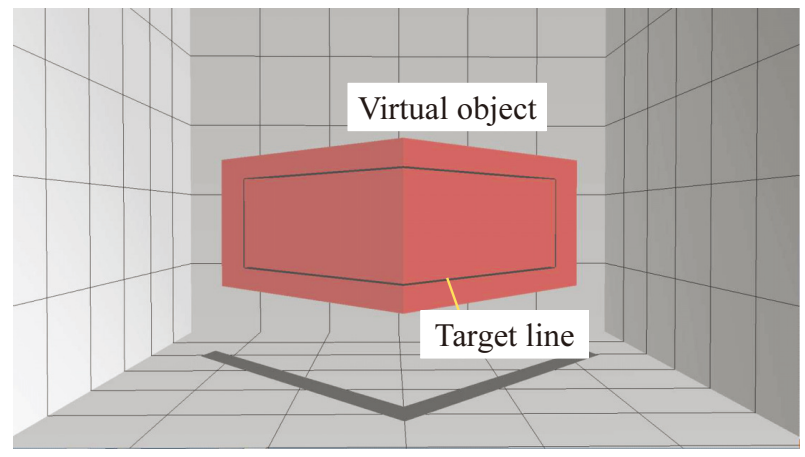

Fig. 15 Test object (cuboid).

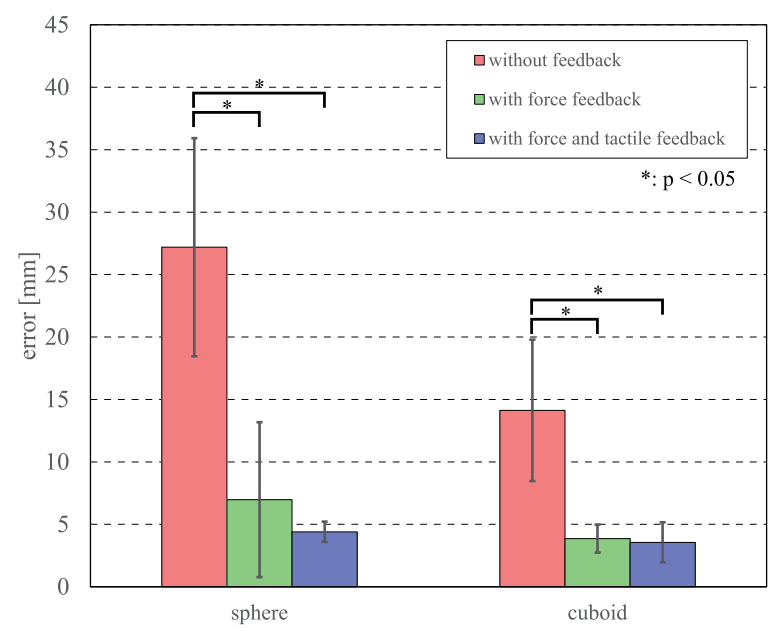

Fig. 16 Average error and standard deviation of each condition.

The results show that $R$ was maximized in trials without feedback. When the subjects took part in a trial without feedback, the virtual tip of the stylus penetrated the surface of the virtual object. Therefore, it was difficult for the subject to point the stylus in the intended direction. On the other hand, when the subject took part in a trial with force feedback, the virtual tip of the stylus did not penetrate the surface of the virtual object due to the force from the virtual object, and the subject was able to trace the target line. Additionally, in a trial with tactile feedback, the subject was able to experience a tactile sensation when the virtual tip of the stylus disengaged from the target line. Thus the subject was able to understand whether the virtual tip of the stylus was touching the target line not only visually but tactilely. In the trials with both force feedback and tactile feedback, the error was the minimized.

\section{3 Discussion}

The objective experimental results show that control of the system without feedback is difficult in the proposed system, and that control with haptic sensation is easier than without feedback. The proposed system

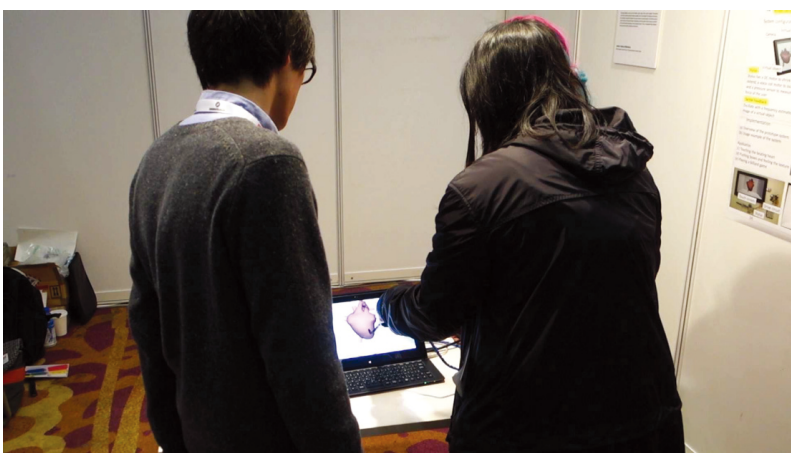

Fig. 17 Scene of a demonstration at ACM SIGGRAPH Asia 2014 Emerging Technologies.

provides only force in the direction of the shrinkage of the stylus. Therefore, a user cannot touch a virtual object in a motion direction parallel to the touch panel. However, the user can change the orientation of the touch panel and touch the virtual object from that direction. Summarizing the above, a user can only feel the force in the direction of the shrinkage of the stylus, however, the user can touch a virtual object from any direction.

The system has the merit that it does not need to be fixed in place. Therefore, the system can be used anywhere and the user only needs a touch panel and a retractable stylus. In future work, we would like to develop the system so that it can show a virtual object that is overlaid on a real image taken by a rear camera, and for the user to be able to touch this virtual object in real space. The system provides the haptic sensation of a real environment as well as the virtual object by using the method described in Section 4. Therefore, the system allows the augmentation of both a virtual object and a real object.

We did not measure the force value the user receives when using this system. To give the user a more realistic sensation from a virtual object, it is necessary to measure the force.

\section{4 Demonstrations}

We had a demonstration of the proposed system at ACM SIGGRAPH Asia 2014 ${ }^{12)}$, an international conference on computer graphics and interactive techniques. More than 6,000 persons attended the conference, and over 100 persons tried our proposed system. Figure 17 shows a scene of the demonstration.

\section{Conclusions}

In this paper, we proposed a system for touching a virtual object behind a mobile touch panel using a retractable stylus. Using this system, the user receives 
the sensation of touching a virtual object behind a mobile touch panel. Experimental results with seven subjects show that the subjects were able to experience the sensation of touching objects behind the mobile touch panel.

The system proposed in this paper is not only a system that provides a haptic sensation, but one that will offer a novel mobile touch panel interface in the future. For example, the system can show a picture taken at a remote location in real time, and the user can touch and feel objects in the picture taken at the remote location. In addition, this system is applicable as an interface for remote machines or surgical robots.

\section{References}

1) G.C. Burdea, "Force and touch feedback for virtual reality", Wiley-Interscience, 1996

2) R. Azuma, Y. Baillot, R. Behringer, S. Feiner, S. Julier and B. MacIntyre, "Recent advances in augmented reality", IEEE Computer Graphics and Applications, vol. 21, no. 6, pp. 34-47, 2001.

3) D.R. Begault, "3-D sound for virtual reality and multimedia", Academic Press, 1994.

4) M. Sato, "SPIDAR and virtual reality", 2002 Proceedings of the 5th Biannual World Automation Congress, vol. 13, pp. 17-23, 2002.

5) T.H. Massie and J.K. Salisbury, "The PHANTOM haptic interface: A device for probing virtual objects", Proceedings of the ASME Winter Annual Meeting, Symposium on Haptic Interfaces for Virtual Environment and Teleoperator Systems, 1994.

6) G. Nikolakis, D. Tzovaras, S. Moustakidis and M. G. Strintzis, "CyberGrasp and PHANTOM integration: Enhanced haptic access for visually impaired users", SPECOM, Conference Speech and Computer, 2004.

7) J. Lee and H. Ishii, "Beyond: Collapsible tools and gestures for computational design", CHI '10 Extended Abstracts on Human Factors in Computing Systems, pp. 3931-3936, 2010.

8) A. Withana, M. Kondo, Y. Makino, G. Kakehi, M. Sugimoto, and M. Inami, "ImpAct: Immersive haptic stylus to enable direct touch and manipulation for surface computing", ACM Computer in Entertainment, vol. 8, no. 2, pp. 9:1-9:16, 2010.

9) Y. Yokoyama, S. Okabe and K. Ishikawa, "Reduction of kinetic friction by harmoinic vibration in an arbitrary direction", Transactions of the Japan Society of Mechanical Engineers, vol. 36, no. 286, pp. 915-922, 1970.

10) T. Owaki, Y. Nakabo, A. Namiki, I. Ishii and M. Ishikawa, "Realtime system for virtually touching objects in the real world using modality transformation from images to haptic information", Systems and Computers in Japan, vol. 30, issue. 9, pp. 17-24, 1999.

11) P. Viola and M. Jones, "Rapid object detection using a boosted cascade of simple features", Conference on Computer Vision and Pattern Recognition, vol. 1, pp. I-511-I-518, 2001.

12) S. Nagasaka, Y. Uranishi, S. Yoshimoto, M. Imura and O. Oshiro, "Haptylus: Haptic stylus for interaction with virtual objects behind a touch screen", ACM SIGGRAPH Asia 2014 Emerging Technologies, Article no. 9, 2014

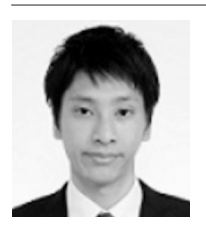

Shingo Nagasaka received the MS degree in engineering from the Graduate School of Engineering Science, Osaka University, Japan in 2015. He is presently with DENSO Corporation. His research interests include human-computer interaction.

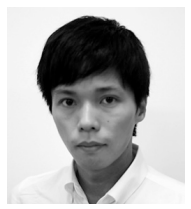

Yuki Uranishi received the $\mathrm{PhD}$ degree in engineering from the Graduate School of Information Science, Nara Institute of Science and Technology, Japan in 2008. He is an assistant professor of the Division of Medical Information Technology and Administration Planning at Kyoto University Hospital in Japan. Before joining Kyoto University Hospital, he was an assistant professor at Nara Institute of Science and Technology from 2009 to 2012 and at Osaka University from 2012 to 2014. His research interests include three-dimensional measurement, augmented reality and human-computer interaction.

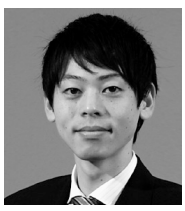

Shunsuke Yoshimoto received the PhD degree in engineering from the Graduate School of Engineering Science, Osaka University, Japan in 2012. Since 2012, he has been an assistant professor in Graduate School of Engineering Science, Osaka University, Japan. His research interests include haptic interaction technologies and biomedical instrumentation.

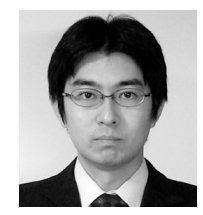

Masataka Imura received the $\mathrm{PhD}$ degree in engineering from the Graduate School of Information Science, Nara Institute of Science and Technology, Japan in 2001. He is a professor in the School of Science and Technology, Kwansei Gakuin University, Japan. Before joining Kwansei Gakuin University, he was an assistant professor at Nara Institute of Science and Technology from 2001 to 2009 and he was an associate professor at Osaka University from 2009 to 2015. His main research interests include virtual reality technology, computer graphics, and medical image processing.

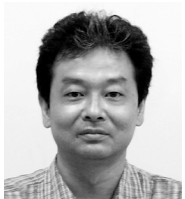

Osamu Oshiro received the $\mathrm{PhD}$ degree in engineering from the Graduate School of Engineering Science, Osaka University, Japan in 1990 $\mathrm{He}$ is a professor in the Graduate School of Engineering Science, Osaka University, Japan. Before joining Osaka University, he was a research engineer at Sumitomo Metal Industries from 1990 to 1993 and he was an assistant/associate professor at Nara Institute of Science and Technology from 1993 to 2003. His research interests include medical imaging and biomedical engineering. 